\title{
A QTL associated with high seed coat cracking rate of a leading Japanese soybean variety
}

\author{
Masayasu Saruta ${ }^{1,2)}$, Yoshitake Takada ${ }^{1)}$, Ken-ichiro Yamashita ${ }^{1)}$, Takashi Sayama ${ }^{1)}$ and Kunihiko Komatsu*1) \\ 1) Western Region Agricultural Research Center Shikoku Research Station, National Agriculture and Food Research Organization, 1-3-1 \\ Sen-yu, Zentsuji, Kagawa 765-8505, Japan \\ 2) Present address: Institute of Crop Science, National Agriculture and Food Research Organization, 2-1-2 Kannondai, Tsukuba, Ibaraki \\ 305-8602, Japan
}

Seed coat cracking in soybeans [Glycine $\max (\mathrm{L})$. Merr.] leads to commercial and agronomic losses. The Japanese elite soybean cultivar 'Fukuyutaka' is often used as a parent for breeding, but its high rate of seed coat cracking is an obstacle to its further use in breeding programs. To establish a DNA marker-assisted selection system for seed coat cracking, genetic factors related to high rates of seed coat cracking were surveyed, and a quantitative trait locus (QTL) with a stable effect on seed coat cracking in both years of a two-year replication experiment was detected on chromosome 20. Comparison of a set of near-isogenic lines (NILs) around this locus verified that the presence of the 'Fukuyutaka' allele significantly increased seed coat cracking in the kernel. The locus is located in a genomic region spanning 3.2 Mb. Marker-assisted selection for the locus will improve the selection efficiency of 'Fukuyutaka'-derived breeding populations.

Key Words: composite interval mapping, cultivar 'Fukuyutaka', DNA marker, near-isogenic line, residual heterozygote line, seed coat defect.

\section{Introduction}

Seed coat cracking (SCC) in soybean harms producers both commercially and agriculturally for the following reasons. First, SCC spoils the appearance of soybean seed and sometimes affects bean curd (tofu) yield and quality (Hou and Chang 1998), thereby causing price decline. Second, SCC affects the germination and vigor of seed (Burchett et al. 1985, Thomison et al. 1989). Soybean SCC rate varies with both environmental conditions and the genetic background of the cultivar (Burchett et al. 1985, Okabe 1996). As cultivation methods to avoid cracking have not been established, genetic improvement to reduce SCC is very important.

The soybean cultivar 'Fukuyutaka' ('JP29668' in the Genebank Project, National Agriculture and Food Research Organization) is superior to other cultivars in yield and suitability for bean curd (tofu) production (Toda et al. 2003). Because of these advantages, 'Fukuyutaka' is utilized as a parent to develop new cultivars in Japan and even in the United States (Carter et al. 2010, 2015). However, it is em-

Communicated by Toyoaki Anai

Received June 11, 2019. Accepted August 22, 2019.

First Published Online in J-STAGE on October 25, 2019.

*Corresponding author (e-mail: kkomatsu@affrc.go.jp) pirically known that some portion of 'Fukuyutaka' progeny tend to show a high SCC rate, leading to their rejection during breeding selection. As the SCC rate is easily influenced by environmental conditions, evaluation and selection are generally repeated over several years. To improve SCC selection efficiency in breeding, DNA markers would be an effective tool.

Molecular marker-based genetic analyses on soybean SCC have already been conducted, and some quantitative trait loci (QTLs) have been reported. Oyoo et al. (2010a) detected two QTLs on linkage group C2 (chromosome 6) from a Japanese landrace. Two other QTLs located on linkage group D1b (chromosome 2) and M (chromosome 7) were detected in a Japanese cultivar (Oyoo et al. 2010b). Ha et al. (2012) identified a total of 12 QTLs with additive effect, two pairs of epistatic QTLs, and epistasis-by-environment interactions. Although DNA markers flanking the QTLs should be effective for progenies of lines or cultivars used in the analyses, it is still unclear whether the markers are effective for SCC selection of 'Fukuyutaka' progeny. In this study, we attempted to detect QTLs associated with the high SCC rate of 'Fukuyutaka' and confirm their effects. 


\section{Materials and Methods}

Plant materials and SCC rate evaluation for QTL analysis

A set of recombinant inbred lines (RILs) developed from a cross of cultivars 'Fukuyutaka' and 'Akimaro' (registered to the Genebank Project, National Agriculture and Food Research Organization; however, the registration number has not yet been announced) was used for QTL analysis. 'Fukuyutaka' is a leading Japanese variety with relatively high SCC rate (Fig. 1). 'Akimaro' is a cultivar adapted to the western region of Japan that exhibits low SCC rate (Fig. 1). The RIL population consisted of $172 \mathrm{~F}_{2}$-derived lines developed using the single seed descent method. SCC rate was evaluated at the $\mathrm{F}_{7}$ and $\mathrm{F}_{8}$ generations.

The RILs were planted to experimental fields at the National Agriculture and Food Research Organization, Western Region Agricultural Research Center, Shikoku Research Station (NARO-WRARC-SRS) at Zentsuji City $\left(34^{\circ} 13^{\prime} \mathrm{N}\right.$, $133^{\circ} 46^{\prime} \mathrm{E}$ ), on June 20, 2014 and June 22, 2015. Around seven plant individuals per inbred line were grown in both years. Each plot was $1 \mathrm{~m}$ long and $70 \mathrm{~cm}$ wide, and the space between plants in each row was around $13 \mathrm{~cm}$. The seeding period and planting density followed the soybean cropping custom of the region. After maturation, four or five plants located at the inner position of each plot were harvested, and seeds of the plants were collected. Four hundred kernels per line were randomly extracted from the collected seeds, and the SCC of each kernel was checked. Even a pinhole on the seed coat that seemed to be a spontaneous seed coat split was considered as SCC. Based on the results, the percentage of SCC kernels was calculated for each line.

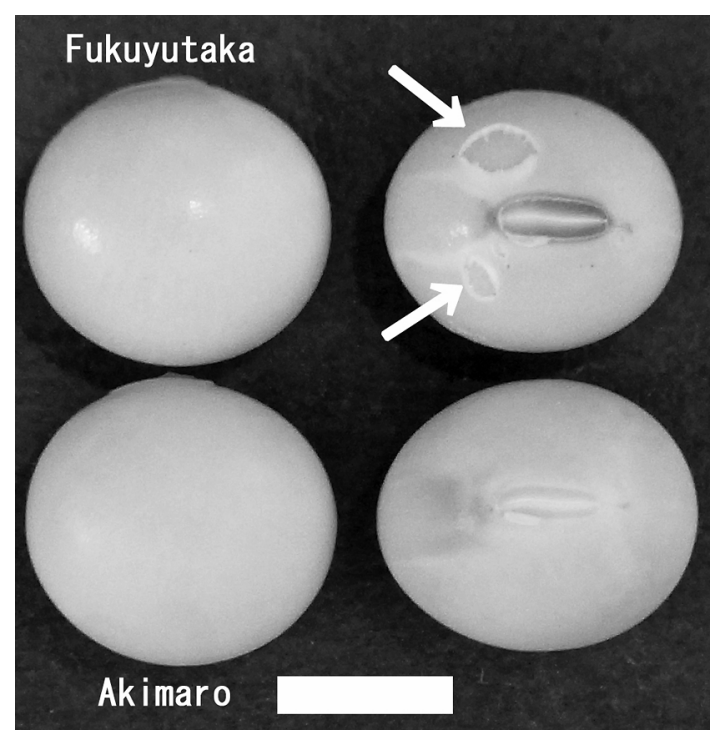

Fig. 1. Seed appearance of the cultivars 'Fukuyutaka' and 'Akimaro'. The two cultivars were used as parents to construct a segregating population for the QTL analysis. Arrows indicate cracks on the seed coats of 'Fukuyutaka'. Scale bar $=5 \mathrm{~mm}$.

\section{QTL analysis of kernel SCC rate}

A linkage map was constructed from the genotype data of 264 simple sequence repeat (SSR) loci (Supplemental Table 1) from the RIL population. DNA was extracted from leaf tissue of each line at the $\mathrm{F}_{6}$ generation in 2013, using a slightly modified version of the method of Edwards et al. (1991). The modification was the exclusion of SDS from the original components of the extraction buffer. DNA fragments corresponding to the SSR loci were amplified with a GeneAmp PCR 9700 System (Thermo Fisher Scientific, Waltham, MA, USA) using the DNA polymerase reagent AmpliTaq Gold 360 mix (Thermo Fisher Scientific, USA). Time and temperature conditions for amplification were as follows: 10 min preheat at $95^{\circ} \mathrm{C}, 35$ three-step cycles of $30 \mathrm{sec}$ denaturation at $95^{\circ} \mathrm{C}, 30 \mathrm{sec}$ annealing at $47^{\circ} \mathrm{C}$, and $30 \mathrm{sec}$ extension at $67^{\circ} \mathrm{C}$, with a final extension at $67^{\circ} \mathrm{C}$ for $7 \mathrm{~min}$. PCR products were treated with an automatic electrophoresis machine, LabChip GX Touch (PerkinElmer, Waltham, MA, USA), to check the fragment size. For some loci, the resultant products were electrophoresed manually in $10 \%$ acrylamide gel and stained with GelRed solution (FUJIFILM Wako Pure Chemical, Osaka, Japan). The genotype data was processed with MAPMAKER/EXP $3.0 \mathrm{~b}$ software (Lincoln et al. 1992) to construct a genetic linkage map. The mapping function used to calculate linkage distance of the SSR loci was Kosambi, and the logarithm of odds (LOD) threshold for linkage establishment was 3.0. The composite interval mapping method (Zeng 1993, 1994) of QTL Cartographer 2.5 (Wang et al. 2012) software was used to detect QTL(s). An LOD threshold equivalent to a 5\% type I error rate for QTL detection was estimated with a $1000 \times$ permutation test (Churchill and Doerge 1994).

\section{Confirmation of detected QTL effects}

A QTL on chromosome 20 was detected in the genetic analysis described above, but the locus was estimated using statistical methods, and thus its existence was not directly proven. Definite evidence showing the effect of the detected QTL was necessary for further analysis, as the SCC rate is influenced by environmental conditions. To verify the effects of genetic factors like QTLs, it is reasonable to compare near-isogenic lines (NILs) that differ only at the genome region of the locus. For the NIL comparison analysis, the residual heterozygote line (RHL) is useful, as a pair of NILs can be easily obtained from its inbred posterity seeds (Tuinstra et al. 1997, Yamanaka et al. 2005). Based on the results of our QTL analysis and genotyping data, RHLs in the RIL population were surveyed and a candidate line, 'FA\#87', was discovered. With additional SSR loci (Supplemental Table 2), the detailed genomic structure around the detected QTL of 'FA\#87' was clarified. After certification that the 'FA\#87' was truly heterozygous around the QTL region, seeds fixed for the 'Fukuyutaka' haplotype and 'Akimaro' haplotype were surveyed. DNA was extracted from the inbred seed fraction of 'FA\#87', and genotypes of the additive SSR loci around the QTL were checked. A 
DNeasy Plant Kit (QIAGEN, Hilden, Germany) or GenCheck DNA Extraction Reagent (Fasmac, Kanagawa, Japan) were used for the extraction. PCR conditions followed a report by Song et al. (2010). The electrophoresis procedure for the PCR products was the same as the aforementioned method for QTL analysis. Inbred seeds fixed for the 'Fukuyutaka' haplotype (hereafter 'FA\#87-Fu') and 'Akimaro' haplotype (hereafter 'FA\#87-Ak') were planted in an experimental field at NARO-WRARC-SRS on July 11, 2017. The planting pattern was same as the RIL cultivation for QTL analysis described above, except that the plot was lengthened to $3 \mathrm{~m}$. Around 20 plants, excluding bordering ones, were harvested, and their seeds were used for subsequent evaluation of SCC. In addition to the field experiments, 'FA\#87-Fu' and 'FA\#87-Ak' were also grown in a greenhouse at NARO-WRARC-SRS in 2018. Plants of both lines were grown in plastic pots $(220 \mathrm{~mm}$ diameter, $300 \mathrm{~mm}$ tall) filled with Tsuchi-Taro potting compost (Sumitomo Forestry Landscaping, Tokyo, Japan). The seeding date was June 28, and around 20 plants for each line were harvested. All the obtained bean kernels were checked, and the SCC rate was calculated for each individual plant.

\section{Results}

\section{SCC rate of the RIL population}

The frequency distribution of the SCC rate of the RIL population and its parents is shown in Fig. 2. 'Fukuytaka' exhibited a higher SCC rate as compared to 'Akimaro' in both years. Frequency of the lowest SCC rate class (under $10 \%$ ) was the highest in both years, and frequency generally decreased as the rate increased. The distributions were estimated to deviate from normal distribution at the $0.1 \%$ level by the Shapiro-Wilk test (data not shown). Correlation coefficients between the 2014 and 2015 trials were 0.79 and were statistically significant at the $0.1 \%$ level. The yearly relationship of the RIL SCC rate is shown as a scatter diagram (Fig. 3).

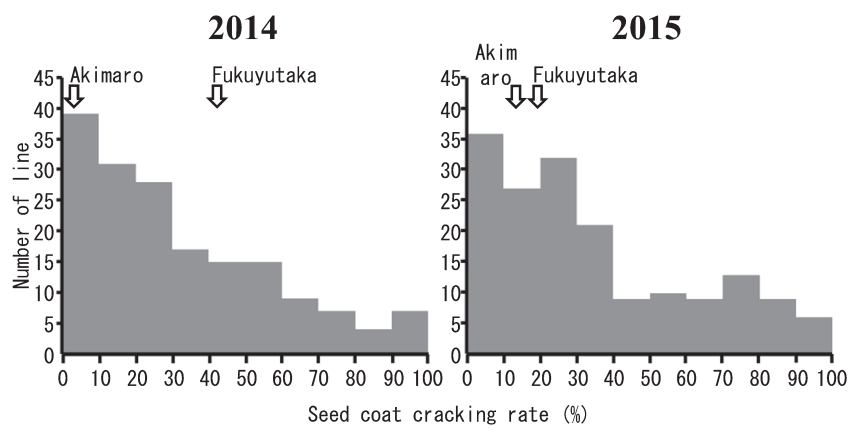

Fig. 2. Frequency distribution of seed coat cracking rate of a recombinant inbred population derived from 'Fukuyutaka' and 'Akimaro'. Arrows above the columns indicate cracking rate of the parental cultivars of the population.

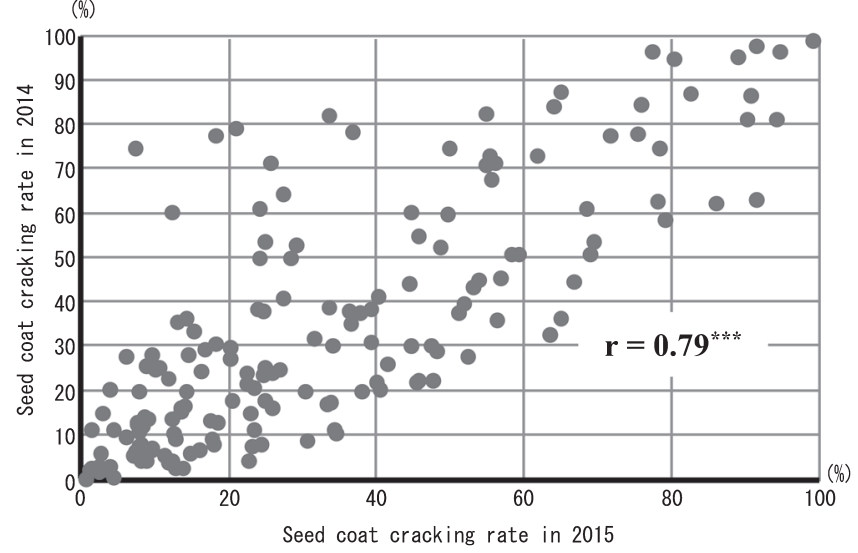

Fig. 3. Year over year relationship of seed coat cracking rate of the recombinant inbred population derived from 'Fukuyutaka' and 'Akimaro'. The three asterisks on correlation coefficient (r) indicate that the value is statistically significant at the $0.1 \%$ level.

Table 1. QTLs for seed coat cracking rate detected in an RIL population derived from 'Fukuyutaka'

\begin{tabular}{cclcrr}
\hline \hline Year & $\begin{array}{c}\text { Chromosome } \\
\text { number }\end{array}$ & Marker interval & LOD $^{a}$ & $\begin{array}{c}\text { Additive } \\
\text { effect }\end{array}$ & $\begin{array}{r}r^{2 c} \\
(\%)\end{array}$ \\
\hline 2014 & 20 & Sat_219-Satt614 & 3.4 & -6.8 & 7.1 \\
& 20 & Sat_418-Satt330 & 4.5 & 8.1 & 9.5 \\
& 20 & Sat_421-Sat_155 & 3.7 & 8.0 & 9.8 \\
\hline 2015 & 10 & Satt592-GMES4019 & 9.8 & -12.7 & 21.5 \\
& 20 & Sat_418-Satt330 & 3.8 & 7.4 & 7.1 \\
\hline
\end{tabular}

${ }^{a}$ Logarithm of odds. LOD score of 3.2 in 2014 trial and 3.3 in 2015 trial are equivalent to a $5 \%$ level of type I error rate.

${ }^{b}$ Effect of 'Fukuyutaka' allele.

${ }^{c}$ Portion of variance explained by the locus.

\section{QTLs associated with kernel SCC rate}

The genetic linkage map constructed from the genotype information of the RIL population spanned $2519.2 \mathrm{cM}$ and covered all 20 chromosomes well (Supplemental Table 1). The sequential order of the loci in the linkage groups (chromosomes) and linkage distances among them were similar to those in previous reports (Cregan et al. 1999, Sayama et al. 2011, Song et al. 2004). The results indicated that the map was suitable for subsequent QTL analysis. Results of composite interval mapping to detect QTLs for SCC rate are shown in Table 1. Three LOD peaks in 2014 and two peaks in 2015 were significant at the 5\% level and were thus regarded as QTLs. In particular, the LOD peak on the central position of chromosome 20 was stably significant throughout the two trials (Fig. 4A). Therefore, we considered this locus to be a genetic factor for SCC and named it quantitative trait locus of the $\underline{\text { Seed coat }}$ cracking rate on chromosome 20, number 1 (qScr20-1). We estimated that the 'Fukuyutaka' allele of $q S c r 20-1$ increased the SCC rate (Table 1). Based on these results, subsequent tests to verify the effects of QTLs were designed only for qScr20-1.

Verification of the effect of the detected QTL

NILs ('FA\#87-Fu' and 'FA\#87-Ak') derived from 
A

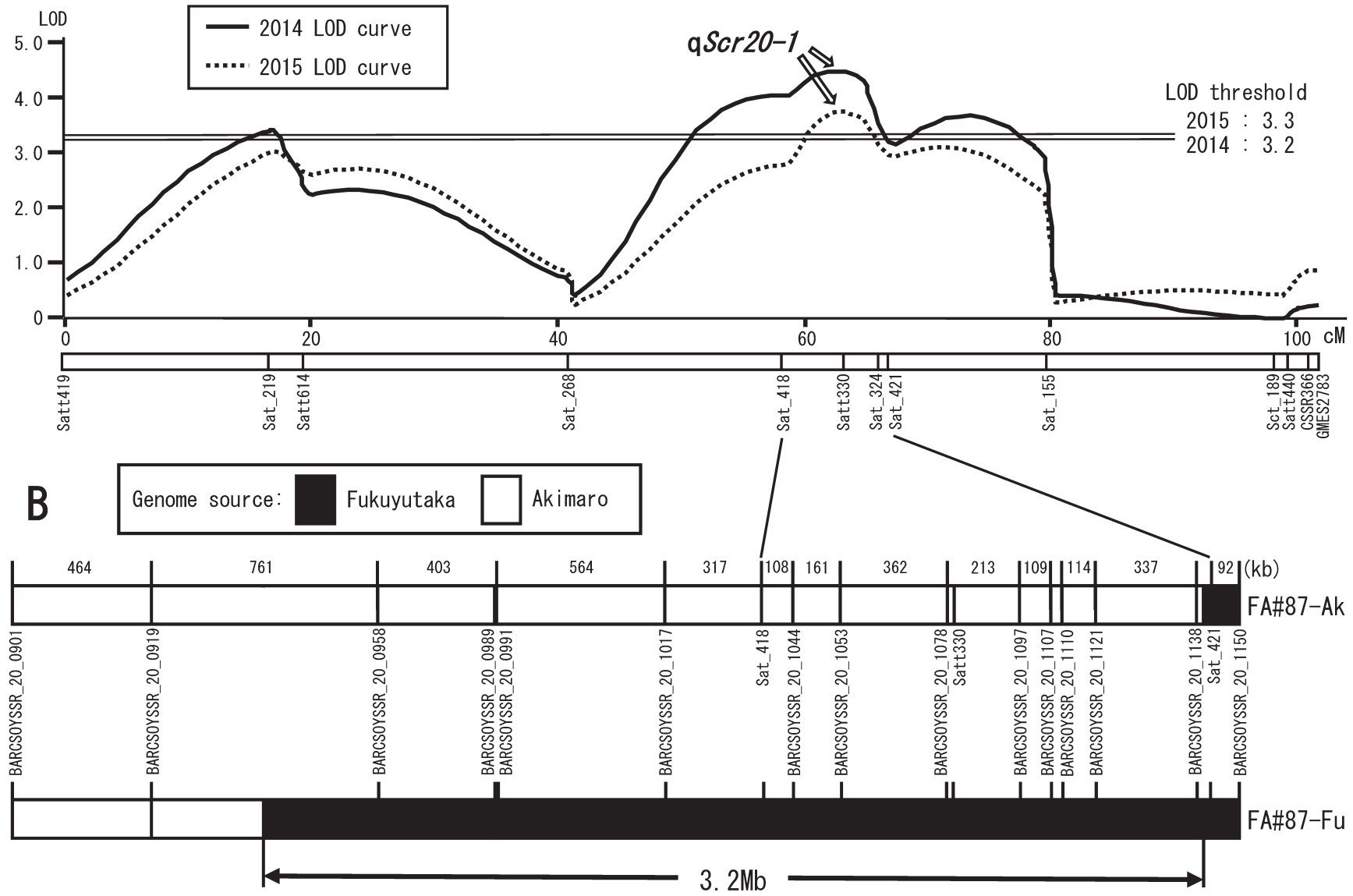

Fig. 4. Detected QTL ( S Scr20-1) for seed coat cracking rate and graphical genotypes of a set of near-isogenic lines about the QTL derived from residual heterozygous line 'FA\#87'. Relationships of LOD curves of seed coat cracking rate, position of the QTL, and genotypes of the near-isogenic lines are shown. A: LOD curves of seed coat cracking rate in 2014 and 2015 trials are shown as solid and dotted lines, respectively. The LOD peak that was significant in both years is considered a firm QTL and named qScr20-1. The horizontal bar at the bottom of the LOD graph is the image of the chromosome. Positions and names of marker loci are shown in the image. The distances among the marker loci are based on recombination frequency in the population used in this study. B: Thick horizontal bars in white and black indicate the image of the chromosome of the near-isogenic lines. The black and white sections indicate genome blocks originating in cultivars 'Fukuyutaka' and 'Akimaro', respectively. SSR marker loci are indicated as vertical lines on the chromosome images, and the names are shown between the depicted chromosomes. The distances among the SSR loci are based on physical distances.

'FA\#87' were confirmed to be almost genetically identical, except for the genomic region where $q$ Scr20-1 is located (Fig. 4B). The width of the different genomic region was estimated to be around $3.2 \mathrm{Mb}$, following BLAST search results of SSR primer sequence in the 'Glycine max Wm82.a2.v1' assembly published on the Phytozome v.12 website (https:// phytozome.jgi.doe.gov/pz/portal.html\#, verified on April 18, 2019). The NILs exhibited statistically significant differences in SCC rate under both field and greenhouse conditions (Fig. 5). In both trials, 'FA\#87-Fu' exhibited significantly higher SCC rate, even though the numerical values varied between trials.

\section{Discussion}

The SCC rate of the RIL population exhibited high correla- tion coefficients for the two years' trials in 2014 and 2015. This suggests that some genetic factor(s) is strongly related to the wide variation in the kernel SCC rate, and thus the phenotype data is suitable for subsequent genetic analysis. In fact, a QTL with stable effect throughout the two-year study was detected on chromosome 20 . The effect was confirmed with NIL comparison tests under two different environmental conditions. In all cases, the 'Fukuyutaka' allele of the locus raised the kernel SCC rate. Based on these results, we regarded the QTL qScr20-1 as one of the genetic factors associated with the high kernel SCC rate of 'Fukuyutaka'.

In the analysis of the NILs for $q S \operatorname{Sc} 20-1$, there was a remarkable difference in the SCC rates between field- and greenhouse-grown plants. Both of the qScr20-1 NILs FA\#87-Fu and FA\#87-Ak exhibited significantly higher SCC rates when greenhouse grown than when open field 

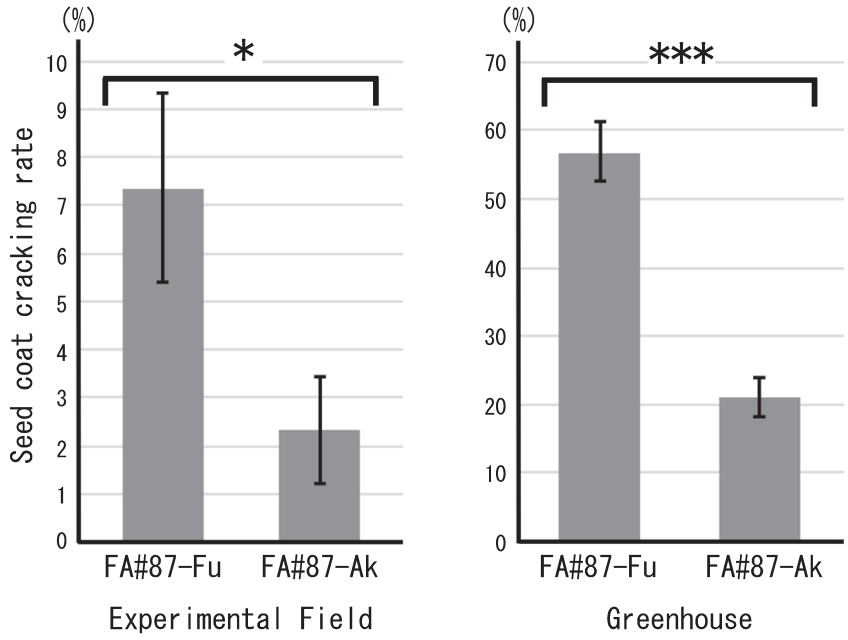

Fig. 5. Seed coat cracking rate of a set of near isogenic lines of qScr20-1 under two different environments. The vertical bar at the top of each column indicates standard error. Statistical significance at the $5 \%$ level and $0.1 \%$ level for 'FA\#87-Fu' and 'FA\#87-Ak' is symbolized as '*' and '***', respectively.

grown $(P<0.001)$. It has been reported that the environmental conditions during the maturation period can influence SCC (Suzuki et al. 1979). According to this report, hot and dry conditions can lead to SCC. Thus, it is quite likely that the hotter and drier climate in the greenhouse compared with that in the open field increased the rate of SCC since the analysis was carried out in the summer and early fall.

No other locus for SCC has been detected around the chromosomal region of $q S \mathrm{~S} 20 \mathrm{2}-1$ thus far; however, QTLs or genetic factors on same chromosome were reported by Ha et al. (2012) and Woodworth and Williams (1938). The LOD peak of the locus in Ha's report is more than $40 \mathrm{cM}$ from the LOD peak of $q S c r 20-1$; thus, they appear to be independent. As for the locus reported by Woodworth and Williams, its position on the chromosome is not clear as the analysis was carried out before molecular genetic markers became available. It is possible to estimate that the locus is located on chromosome 20 from genetic linkage with the genes for other traits. According to the report, the locus for SCC (called De2) is tightly linked with the $P 2$ locus for minute pubescence. $P 2$ has been reported to be located on 'Linkage Group I' (Cregan et al. 1999), and this linkage group was assigned to chromosome 20 (Zou et al. 2003). Unfortunately, it is impossible to clarify the relationship of qScr20-1 and De2 based on the results to date. Further study on allelism of the loci on chromosome 20 might be required for a better understanding of SCC rate control.

Based on the results of the QTL analysis, the impact of $q S c r 20-1$ on SCC rate is not necessarily high. It is quite difficult to explain the wide kernel SCC rate variation in the RIL population using the locus alone. However, we think this information on $q S c r 20-1$ is still valuable and useful for soybean breeders as it makes it possible to omit breeding lines that possess a risk factor for high kernel SCC rate.
DNA marker-assisted selection makes it possible to reduce experimental field acreage and labor for SCC selection by half. We tackled an analysis to determine the accurate chromosomal position of the qScr20-1 locus to implement a DNA marker-assisted selection system for SCC into our breeding programs.

In addition to research on SCC being directly applicable for breeding, studies on genetic or physiological causes of SCC occurrence are also important, as comprehension of the cause may contribute to the appropriate choice of parents to breed cultivars with less SCC, as well as to development of more powerful DNA marker-assisted selection systems effective for breeding populations with a wider genetic background. Despite this, few analytical progresses have been reported. Senda et al. (2017) suggested that proanthocyanidins and lignin are related to the SCC of buff-pigmented soybean seeds. Percy et al. (1999) reported that soluble proline-rich cell wall protein is absent in net-like seed-coat-defective soybean lines. The phenomenon was estimated to be related directly to severe SCC and caused by certain posttranslational events, like rapid cross-linking into the cell wall matrix. Comparing the net-like SCC isogenic lines, Kour et al. (2014) revealed that 364 genes are differentially expressed in the seed coat at the middle developmental stage. It was also revealed that $19 \%$ of the differentially expressed genes are related to cell wall developmental processes, such as cell wall proline-rich and glycine-rich cell wall protein genes. Even these SCC-related physiological discoveries are quite interesting and important but are not adequate to clarify the mechanism of SCC occurrence. Therefore, other analytical attempts from different perspectives and using different research materials are necessary. The results of our study and plant materials such as RHL-derived NILs might be stepping stones for further analysis.

This study showed that one or more genetic factors associated with a high rate of SCC in 'Fukuyutaka' are located in a $3.2 \mathrm{Mb}$ region of chromosome 20. According to the 'Glycine max Wm82.a2.v1' assembly on the Phytozome website (https://phytozome.jgi.doe.gov/pz/portal.html\#, verified on July 17, 2019), this genome region contains some interesting genes that may be related to the mechanism underlying SCC. Around the LOD peak of $q S c r 20-1$, there are three genes that are predicted to be related to cell morphogenesis or cell wall properties (Glyma.20G158900: an Actin_depolymerizing_factor, Glyma.20G159200: Beta tubulin, and Glyma.20G159900: Pectin_acetylesterase and_similar_proteins). In Arabidopsis, a knockout mutant of actin depolymerizing factor 4 (ADF4) has a longer hypocotyl and longer epidermal cells than the wild type (Henty et al. 2011). Beta tubulin is a component of microtubules, which are a key part of the cytoskeleton that is essential for various cellular functions (Hashimoto 2015). An Arabidopsis double mutant of the pectin acetylesterase genes PAE8 and PAE9 exhibits high pectin acetate content and short inflorescence stems when compared to wild type plants 
(de Souza et al. 2014). The effects of these genes or their orthologs on SCC has not been reported. However, it is likely that malfunction of one or more of these genes would induce structural changes in the cytoskeleton or alter the cell wall properties, leading to a difference in the SCC rate. Additional studies, including positional cloning, along with sequence and expression analyses of candidate genes, would be an effective approach to clarify this hypothesis. Our attempt to determine the accurate chromosomal position of the $q S \operatorname{se} 20-1$ locus might also help elucidate the cause of SCC.

\section{Author Contribution Statement}

M.S.: Development of the RIL population, evaluation of SCC rate of the RILs, genotyping of the RILs, QTL analysis of the SCC rate

Y.T.: Development of the RILs population, genotyping of the RILs, evaluation of SCC rate of the NILs

K.Y.: QTL analysis of the SCC rate, evaluation of SCC rate of the NILs

T.S.: QTL analysis of the SCC rate, evaluation of SCC rate of the NILs

K.K.: QTL analysis of the SCC rate, development and detailed genotyping of the NILs, evaluation of SCC rate of the NILs

\section{Acknowledgments}

The authors express deep gratitude to all support members related to this study for their dedicated work. This study was partly supported by NARO-WRARC original funding for initiation of the research.

\section{Literature Cited}

Burchett, C.A., W.T. Schapaugh,Jr., C.B. Overley and T.L.Walter (1985) Influence of etched seed coats and environmental conditions on soybean seed quality. Crop Sci. 25: 655-660.

Carter,T.E.Jr., P.E.Rzewnicki， J.W.Burton， M.R.Villagarcia， D.T. Bowman, E.Taliercio and P.Kwanyuen (2010) Registration of N6202 Soybean germplasm with high protein, favorable yield potential, large seed, and diverse pedigree. J. Plant Regist. 4: 73-79.

Carter,T.E.Jr., S.M.Todd and A.M.Gillen (2015) Registration of N6002 soybean germplasm with enhanced yield derived from Japanese cultivars Fukuyutaka and Nakasennari and elevated seed protein content. J. Plant Regist. 9: 216-221.

Churchill, G.A. and R.W.Doerge (1994) Empirical threshold values for quantitative trait mapping. Genetics 138: 963-971.

Cregan, P.B., T. Jarvik, A.L. Bush, R.C. Shoemaker, K.G.Lark, A.L.Kahler, N.Kaya, T.T. Vantoai, D.G.Lohnes, J.Chung et al. (1999) An integrated genetic linkage map of the soybean genome. Crop Sci. 39: 1464-1490.

de Souza, A., P.A. Hull, S. Gille and M.Pauly (2014) Identification and functional characterization of the distinct plant pectin esterases PAE8 and PAE9 and their deletion mutants. Planta 240: 11231138.

Edwards, K., C. Johnstone and C. Thompson (1991) A simple and rapid method for the preparation of plant genomic DNA for PCR analysis. Nucleic Acids Res. 19: 1349.

Ha,B.-H., H.-K.Kim and S.-T.Kang (2012) Mapping QTLs with epistatic effects and QTL-by-environment interactions for seed coat cracking in soybeans. Euphytica 186: 933-942.

Hashimoto, T. (2015) Microtubules in plants. Arabidopsis Book 13 $\mathrm{e} 0179$.

Henty, J.L., S.W.Bledsoe, P.Khurana, R.B.Meagher, B.Day, L. Blanchoin and C.J.Staiger (2011) Arabidopsis actin depolymerizing factor4 modulates the stochastic dynamic behavior of actin filaments in the cortical array of epidermal cells. Plant Cell 23: 3711-3726.

Hou, H.J. and S.K.C. Chang (1998) Yield and quality of soft tofu as affected by soybean physical damage and storage. J. Agric. Food Chem. 46: 4798-4805.

Kour,A., A.M. Boone and L.O. Vodkin (2014) RNA-Seq profiling of a defective seed coat mutation in Glycine max reveals differential expression of proline-rich and other cell wall protein transcripts. PLoS ONE 9: e96342.

Lincoln, S., M. Daly and E. Lander (1992) Constructing genetic maps with MAPMAKER/EXP 3.0. Whitehead Institute Technical Report. Whitehead Institute, Cambridge.

Okabe,A. (1996) Inheritance of seed coat cracking and effective selection method for the resistance in soybean. Jpn. Agric. Res. Q. 30: $15-20$.

Oyoo,M.E., E.R.Benitez, H.Matsumura and R.Takahashi (2010a) QTL analysis of seed coat cracking in soybean. Crop Sci. 50: $1230-1235$.

Oyoo, M.E., S.M. Githiri, E.R. Benitez and R. Takahashi (2010b) QTL analysis of net-like cracking in soybean seed coats. Breed. Sci. 60: 28-33.

Percy, J.D., R. Philip and L.O. Vodkin (1999) A defective seed coat pattern (Net) is correlated with the post-transcriptional abundance of soluble proline-rich cell wall proteins. Plant Mol. Biol. 40: 603613.

Sayama,T., T.-Y.Hwang, K.Komatsu, Y.Takada, M.Takahashi, S. Kato, H. Sasama, A. Higashi, Y.Nakamoto, H. Funatsuki et al. (2011) Development and application of a whole-genome simple sequence repeat panel for high-throughput genotyping in soybean DNA Res. 18: 107-115.

Senda, M., N. Yamaguchi, M. Hiraoka, S.Kawada, R. Iiyoshi, K. Yamashita, T. Sonoki, H. Maeda and M. Kawasaki (2017) Accumulation of proanthocyanidins and/or lignin deposition in buffpigmented soybean seed coats may lead to frequent defective cracking. Planta 245: 659-670.

Song, Q.J., L.F. Marek, R.C.Shoemaker, K.G.Lark, V.C.Concibido, A. Delannay, J.E. Specht and P.B.Cregan (2004) A new integrated genetic linkage map of the soybean. Theor. Appl. Genet. 109: 122 128.

Song, Q., G. Jia, Y.Zhu, D. Grant, R.T.Nelson, E.-Y.Hwang, D.L. Hyten and P.B. Cregan (2010) Abundance of SSR motifs and development of candidate polymorphic SSR markers (BARCSOYSSR_1.0) in soybean. Crop Sci. 50: 1950-1960.

Suzuki,M., E. Takahashi and H. Miyakawa (1979) Characteristic of seed coat cracking of soybean and its difference by climatic condition. Tohoku Agric. Res. 25: 59-60.

Thomison, P.R., M.M.Kulik and D.A.Morris (1989) Influence of etched seed coats on Phomopsis infection and electrolyte leakage of soybean seeds. J. Seed Tech. 13: 9-18.

Toda,K., T.Ono, K.Kitamura, M.Hajika, K.Takahashi and Y. Nakamura (2003) Seed protein content and consistency of tofu 
prepared with different magnesium chloride concentrations in six Japanese soybean varieties. Breed. Sci. 53: 217-223.

Tuinstra, M.R., G.Ejeta and P.B. Goldsbrough (1997) Heterogeneous inbred family (HIF) analysis: a method for developing nearisogenic lines that differ at quantitative trait loci. Theor. Appl. Genet. 95: 1005-1011.

Wang, S., C.J.Basten and Z.-B.Zeng (2012) Windows QTL Cartographer 2.5. Department of Statistics, North Carolina State University, Raleigh, NC (http://statgen.ncsu.edu/qtlcart/WQTLCart.htm).

Woodworth, C.M. and L.F. Williams (1938) Recent studies on the genetics of the soybean. J. Am. Soc. Agric. 30: 125-129.

Yamanaka, N., S. Watanabe, K. Toda, M.Hayashi, H.Fuchigami,
R. Takahashi and K. Harada (2005) Fine mapping of the FT1 locus for soybean flowering time using a residual heterozygous line derived from a recombinant inbred line. Theor. Appl. Genet. 110: 634-639.

Zeng,Z.B. (1993) Theoretical basis for separation of multiple linked gene effects in mapping quantitative trait loci. Proc. Natl. Acad. Sci. USA 90: 10972-10976.

Zeng,Z.B. (1994) Precision mapping of quantitative trait loci. Genetics 136: 1457-1468.

Zou, J.J., R.J.Singh, J.Lee, S.J.Xu, P.B.Cregan and T.Hymowitz (2003) Assignment of molecular linkage groups to soybean chromosomes by primary trisomics. Theor. Appl. Genet. 107: 745-750. 